REVIEW

\title{
Best practice in primary care pathology: review 1
}

\author{
W S A Smellie, D Wilson, C A M McNulty, M J Galloway, G A Spickett, D I Finnigan, D A Bareford, \\ M A Greig, J Richards
}

J Clin Pathol 2005;58:1016-1024. doi: 10.1136/jcp.2004.025049

This first best practice review examines four series of common primary care questions in laboratory medicine, namely: (i) measurement and monitoring of cholesterol and of liver and muscle enzymes in patients in the context of lipid lowering drugs, (ii) diagnosis and monitoring of vitamin B12/folate deficiency, (iii) investigation and monitoring of paraprotein bands in blood, and (iv) management of Helicobacter pylori infection. The review is presented in a question-answer format, referenced for each question series. The recommendations represent a précis of guidance found using a standardised literature search of national and international guidance notes, consensus statements, health policy documents, and evidence based medicine reviews, supplemented by MEDLINE EMBASE searches to identify relevant primary research documents. They are not standards but form a guide to be set in the clinical context. Most are consensus rather than evidence based. They will be updated periodically to take account of new information.

See end of article for authors' affiliations

Correspondence to: Dr W S A Smellie, Department of Pathology, Bishop Auckland Hospital General Cockton Hill Road, Bishop Auckland, County Durham DL14 6AD, UK; info@smellie. com

Accepted for publication 17 February 2005

\section{CORONARY PREVENTION (WSAS AND DIF) \\ Lipid/cholesterol management}

Lipid guidelines are changing on a regular basis and the results of this search are current as of May 2005. However, the guidance may change. Most of the guidelines are derived from consensus docu- documents are not fully independent of one another, and there are frequent cross references between documents. Overall agreement is in general close and the minor differences-for example in testing intervals found-are unlikely to have significant relevance to clinical outcome.

However, following the heart protection study results, the decision to make simvastatin available without prescription and the anticipated publication of new joint British societies guidance this year, the summaries below should be seen as the guidance position as of spring 2005, and of all the sets of guidance to be examined is the one that will require updating probably within one year. Target or optimum cholesterol reduction values are not addressed in these questions because of recent changes discussed below. Figure 1 provides an overview of the guidance.

How often should patients' lipids be tested after starting lipid lowering treatment?

We recommend that after starting lipid lowering drug treatment, lipids should be checked:

- Eight $( \pm 4)$ weeks after starting drug treatment.

- Eight $( \pm 4)$ weekly thereafter until on target.

There is close consensus in national and international guidance that patients should have lipid parameters measured after starting statin treatment until a target is reached..$^{2-7}$ The recommended measurement intervals all range between four and 12 weeks after starting drug treatment. The term treatment frequently refers specifically to statins in these documents, although we consider that it is reasonable to extrapolate this to other drugs used in lipid lowering.

The review interval after starting drug treatment will depend on practice resources and logistics, but it is unlikely that differences within this 12 week period will influence longterm outcome.

The heart protection study, ${ }^{8}$ published after the above guidelines, examined a large number of patients with minimal laboratory follow up. However, this was a selected population and it cannot be assumed that tolerability and compliance would be identical in an unselected population. We would expect future guideline ments. Although published separately, these

Abbreviations: $C K$, creatine kinase; DU, duodenal ulcer; GMS, General Medical Services; GU, gastric ulcer; LFT, liver function test; MCV, mean red cell volume; MGUS, monoclonal gammopathy of unknown significance; NICE, National Institute for Health and Clinical Excellence; PPI, proton pump inhibitor; ULN, upper limit of normal 


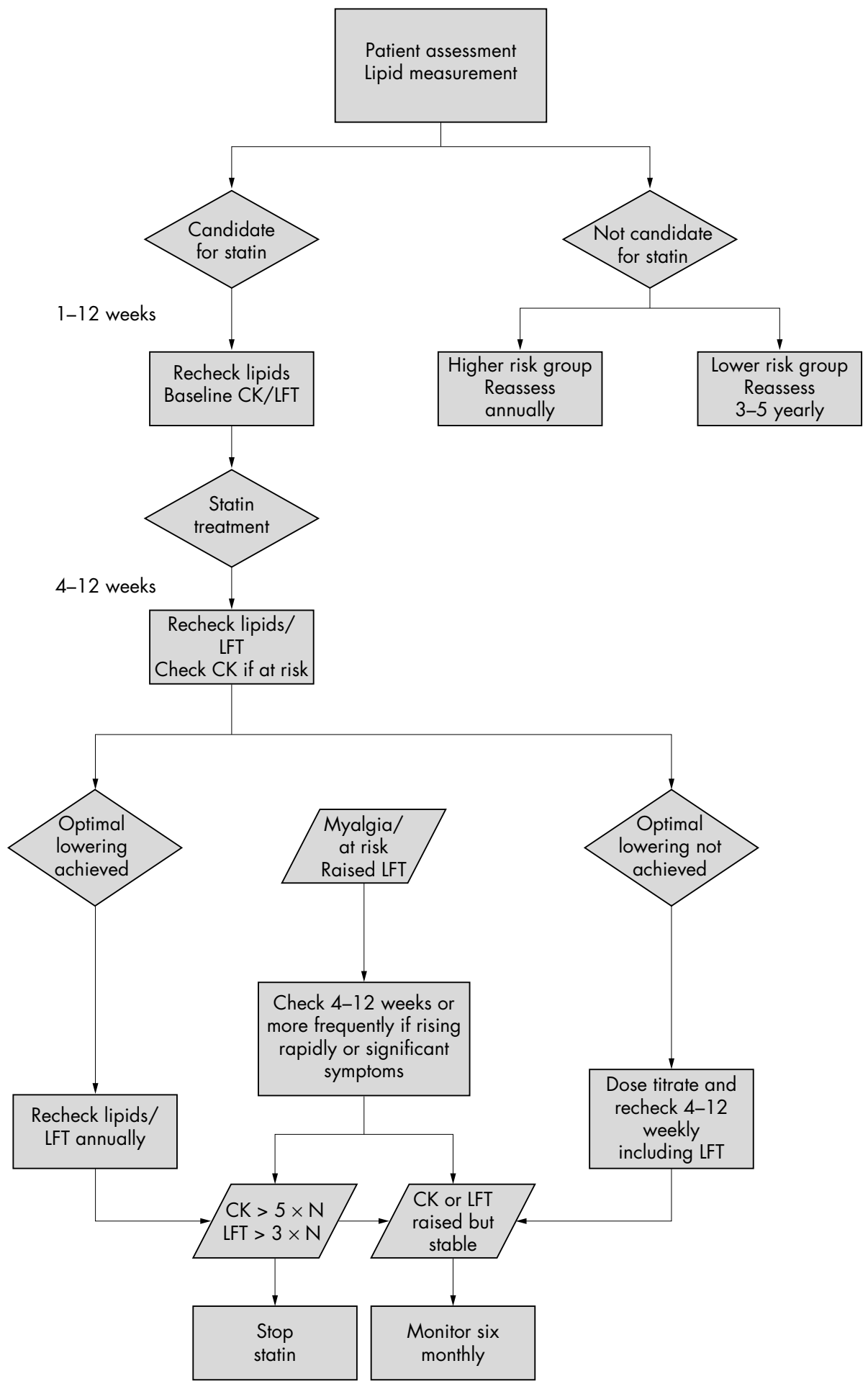

Figure 1 Overview of guidance found for laboratory testing in lipid management. CK, creatine kinase; LFT, liver function test; $\mathrm{N}$, normal.

annually (unless specific reason for more frequent reviews).

There is close consensus in national and international guidelines ${ }^{2-4} 6$ that patients should continue to be monitored once they have reached a target lipid value on lipid lowering treatment. The recommended monitoring interval is most frequently one year. ${ }^{3-5} 7910$

Some guidelines outside of the UK recommend more regular measurements, at intervals of six months ${ }^{6}$ or four to six months ${ }^{2}$ once a patient has reached target. 
The heart protection study, ${ }^{8}$ published after the above guidelines, examined a large number of patients with limited laboratory follow up. Some have therefore questioned the need for laboratory follow up. However, this was a selected population and it cannot be assumed that tolerability and compliance would be identical in an unselected population. We would expect future guideline revisions to consider this option, although it cannot at present be recommended on the basis of a consensus guideline or statement.

GMS contract indicator: percentage of patients with cholesterol result available within 15 months in secondary prevention and diabetes.

How often should cholesterol/lipids be tested when assessing a patient's coronary risk?

We recommend that the decision to start lipid lowering drug treatment should be based on:

- At least two measurements taken one to 12 weeks apart. This is to take account of biological and laboratory variability.

When monitoring risk in patients not receiving lipid lowering drug treatment we recommend:

- In secondary prevention, patients with cholesterol above the treatment start threshold should be tested annually.

- In higher risk primary prevention, patients who have not yet reached the risk threshold for drug treatment should be tested one to two yearly.

- In low risk primary prevention, patients should be tested five yearly.

There is close consensus in both national and international guidance that the decision to start lipid lowering treatment should not be based on just one measurement, but two, ${ }^{346}$ or three. $^{210}$

A recent analysis of the imprecision of coronary risk assessment has suggested that in fact three measurements are needeed to produce a precise estimate of risk. ${ }^{11}$

Although methods used to assess coronary risk in the guidelines vary, all adopt a common approach based on testing at five yearly intervals in the lowest risk groups, ${ }^{35} 91012$ although in Singapore it is every three to five years. ${ }^{6}$ This rises to annually ${ }^{3}{ }^{10}$ or one to two yearly ${ }^{59}{ }^{12}$ in secondary prevention and medium risk primary prevention patients who have yet not reached the threshold for lipid lowering drug treatment.

To use current risk assessment tools, lipid assessments before treatment require total and high density lipoprotein cholesterol (and therefore also potentially at least a calculated density lipoprotein) and triglycerides. Regardless of the risk assessment method used, all of the available guidance recommends these tests.

GMS contract indicator: percentage of patients with cholesterol result available within 15 months in secondary prevention and diabetes.

\section{Liver enzyme management}

How often should liver enzymes be routinely measured in patients taking statins?

We recommend a baseline check before starting treatment with a statin, eight weeks after starting a statin or after any dose increase, then if liver function tests (LFTs) are stable, annual checks thereafter.

There is broad consensus that alanine aminotransferase and/or aspartate aminotransferase should be checked before starting a statin, and then rechecked one to three months after starting treatment. ${ }^{241314}$ There is less agreement on how often LFTs should be measured subsequently. Some authorities recommend only annual checks, ${ }^{13}$ whereas others recommend checks every eight to 12 weeks during the first year of treatment, followed by annual checks thereafter. ${ }^{15}$ The Scottish Intercollegiate Guideline Network recommends a single check after starting and none thereafter if LFTs are normal. Many of the guidelines we reviewed advised or based their recommendations on the monitoring requirements outlined by the manufacturers of the different statins.

Data from the largest statin study to date, the heart protection study, are reassuring. ${ }^{8}$ Over 20000 people were allocated to treatment with a statin or placebo and monitored over five years. The incidence of raised liver enzymes was extremely low, with no excess in the statin treated group. In particular, there was no significant excess in the number of people who had treatment stopped in the statin compared with the placebo group because of raised liver enzymes $(0.5 \%$ $v 0.3 \%)$.

A systematic review of 48 statin trials is similarly reassuring. ${ }^{16}$ There were no cases of liver failure in the trials. Raised liver enzymes occurred in $1.3 \%$ of individuals receiving a statin compared with $1.1 \%$ of those receiving placebo.

On the basis of this, it has been argued that routine monitoring of LFTs is unnecessary in people without preexisting liver disease. This is not in keeping with current monitoring requirements outlined in the product licences, and it is difficult to know how reliably safety data from carefully monitored clinical trials can be generalised to people commonly treated in primary care. However, postmarketing surveillance data are reassuring, suggesting one case of liver failure for each 10 million prescriptions or about one for each million person years of use. ${ }^{16}$

The National Institute for Health and Clinical Excellence (NICE) has recently highlighted the requirement for further data on the need for biochemical monitoring of statins for adverse effects. ${ }^{17}$

GMS contract indicator: none.

What if LFTs become raised in a patient taking a statin?

We recommend that if transaminases become raised in a patient taking a statin:

- If less than three times the upper limit of normal (ULN), continue the statin but recheck LFTs within four to six weeks to exclude further rises in transaminases. If values are stable, no extra monitoring is required.

- If more than or equal to three times greater than the ULN, consider two options (depending on the concentration of transaminase):

- Stop the statin and recheck LFTs within four to six weeks to ensure that values settle. Cautious reintroduction of a statin could be considered at a later date.

- Reduce the dosage of statin and recheck LFTs within four to six weeks. If transaminases continue to be greater than three times the ULN, stop the statin. If transaminases are lower than three times the ULN, a cautious dosage increase could be considered at a later date.

Raised hepatic transaminases occur in $0.5-2.0 \%$ of people taking statins. It is uncertain whether increases in 
transaminases represent true hepatotoxicity. Transaminase values often settle with dosage reduction, and often do not rise again with further increase in dosage or use of another statin. $^{13}$

Most of the guidelines we reviewed recommend stopping statins if transaminases rise to more than three times the ULN. Some suggest that dosage reduction with close monitoring may be an option, but do not provide specific instructions regarding frequency of monitoring. The drug manufacturers generally recommend that if transaminase values become raised, these should be rechecked promptly, and if still raised, monitored closely. If concentrations continue to rise, particularly to above three times the ULN, the manufacturers advise reducing the dose or stopping the drug. Again, no specific advice is given on how frequently monitoring should take place. Our view is that a reasonable interval is four to six weeks.

A recent $B M J$ editorial discussing the criteria that should apply to monitoring drug treatments highlights the difficulty in making clear recommendations regarding monitoring of liver function tests in patients on statins. ${ }^{18}$

GMS contract indicator: none.

Creatine kinase management

How often should creatine kinase (CK) be measured in patients taking statins?

We recommend a baseline check before starting treatment with a statin If the baseline $C K$ value is greater than five times the ULN, do not start a statin.

If a person has no identifiable risk factors for myopathy:

- Routine monitoring of CK is not necessary if taking a statin.

If the person has identifiable risk factors for myopathy (see below):

- Consider carefully the risk/benefit of treatment with a statin.

- If a statin is thought to be necessary then monitoring of $\mathrm{CK}$ is advisable. As a minimum, we recommend a CK check within eight weeks of starting a statin and after any dosage increase.

If a person develops muscle pain, weakness, or cramps while taking a statin:

- Check the CK value as soon as possible.

Myopathy is a rare but potentially serious adverse effect of statin treatment. The incidence of severe myopathy is reported to be $0.08 \%$, and most of these individuals have recognisable risk factors for myopathy. ${ }^{13}$ The CSM $(2002)^{19}$ highlights the following main risk factors:

- Underlying muscle disorders, renal impairment, hypothyroidism, or alcohol abuse.

- Concomitant use of other lipid lowering drugs (fibrates and nicotinic acid).

- Concomitant use of cytochrome P450 3A4 inhibitors, including cyclosporin, macrolide antibiotics (such as erythromycin and clarithromycin), azole antifungal (for example, itraconazole and ketoconazole), and protease inhibitors (such as nelfinavir and indinavir).

Data from the largest statin study to date, the heart protection study, are reassuring. ${ }^{8}$ Over 20000 people were allocated to treatment with a statin or placebo and monitored over five years. Reported muscular pain was no more common in the statin group than in the placebo group, and there was no difference in the number of treatment discontinuations as a result of muscle symptoms $(0.5 \%$ in both groups). However, people were excluded from this trial if they were thought to be at risk of myopathy.

A systematic review of 48 statin trials is also reassuring. ${ }^{16}$ In over 35000 people, with a total of 158000 person years of treatment between them, rhabdomyolysis occurred in eight treated and five placebo patients (none with serious illness or death). A serum CK value greater than 10 times the ULN occurred in 55 treated $(0.17 \%)$ and 43 placebo $(0.13 \%)$ patients.

Postmarketing surveillance data suggest one death from rhabdomyolysis for each 10 million prescriptions, or about one for each million person years of use.

Most guidelines suggest that routine laboratory monitoring of CK is of little value in the absence of clinical symptoms or signs, but that a baseline measurement is helpful to identify asymptomatic CK increases and thereby avoid subsequent confusion. $^{213}$ Therefore, all people receiving statins should be instructed to report muscle discomfort or weakness or brown urine immediately, which should prompt a CK measurement $^{21319}$ and reassessment of the benefit/risk ratio of treatment.

One subgroup of patients who merit specific attention are those of Afro-Caribbean descent, in whom raised CK values of $1000 \mathrm{IU} /$ litre may be seen as a normal variant. No clear guidance exists for the management of this group. The opinion of Heart UK (personal communication, A Wierzbicki, 2005 ) is that this should be treated using a modified threshold to stop treatment of five times the pretreatment value, assuming the patient has no muscle symptoms.

GMS contract indicator: none.

What if CK becomes raised in a patient taking a statin?

We recommend the following if $\mathrm{CK}$ becomes raised in a patient taking a statin.

If more than five times the ULN:

- Stop treatment immediately.

If less than five times the ULN:

- If no muscle symptoms, these patients can usually be treated with a statin. Patients should be alerted to report symptoms carefully and consider further checks of CK to ensure that values are not rising.

- If there are muscle symptoms, follow the patient's symptoms and check CK concentrations regularly (for example, fortnightly) if CK continues to rise. If muscle symptoms are severe or $C K$ values continue to rise, we recommend stopping statin treatment or seeking consultant advice.

Apart from high risk patients (described above) the benefits of statin treatment where indicated are considered to far outweigh the risks. ${ }^{19}$

The $\mathrm{CSM}^{19}$ recommends not starting a statin if the baseline CK is greater than five times the ULN, and stopping the statin if the CK rises to greater than five times the ULN. If the CK returns to normal after stopping the statin, the CSM suggests that the statin may be introduced cautiously at the lowest doses with close monitoring. Management to follow in cases of smaller rises is less clear. Symptoms alone may require the statin to be stopped. If symptoms are not severe and the CK has risen, regular (for example, fortnightly) measurement of $\mathrm{CK}$ to assess the rate of rise would appear prudent. If a patient is symptom free or has mild symptoms and the CK stabilised at a concentration of less than $1000 \mathrm{IU} / \mathrm{litre}$ it is 
often possible to continue the statin, although more regular checks of the CK would appear prudent, even in an asymptomatic patient. There is little guidance on the intervals for checking in such cases, and a period of six monthly is suggested pragmatically.

GMS contract indicator: none.

\section{VITAMIN B 12 AND FOLATE (MJG AND DAB)}

There is limited information from large scale consensus statements or large clinical trials. The guidance below is derived from a small number of reviews supplemented by extrapolations from knowledge of the physiology of vitamin B12 and folate. These answers do not address the further investigation of the cause of vitamin B12 or folate deficiency.

In which patients should vitamin B12 and folate values be measured?

The following are generally agreed indications for the measurement of vitamin B12 and folate concentrations:

- Macrocytic anaemia.

- Macrocytosis (particularly in patients with a mean red cell volume (MCV) above $110 \mathrm{fl}$ ).

- Patients with specific neuropsychiatric abnormalities (vitamin B12 only if no macrocytosis or anaemia).

Note that coexisting conditions such as iron deficiency or thalassaemia trait may mask the development or presence of macrocytosis.

\section{Macrocytic anaemia}

Macrocytosis in itself may or may not be present with anaemia but those patients who have macrocytosis with anaemia should have vitamin B12 and folate measurements performed. ${ }^{20}{ }^{21}$

\section{Macrocytosis}

The level of macrocytosis can predict the probability of vitamin B12 and folate deficiency being present. As the MCV increases above $100 \mathrm{fl}$, so the probability of vitamin B12 and folate deficiency increases. This is particularly true of patients with an MCV above $130 \mathrm{fl}$, except for those receiving hydroxyurea. The probability of B12 deficiency is less with MCVs between 100 and $110 \mathrm{fl}$, which is more likely to be related to other causes of macrocytosis, such as alcohol abuse, liver disease, anti-neoplastic drugs, human immunodeficiency virus infection, and also haematological disorders, such as myelodysplastic syndromes or haemolytic conditions. ${ }^{20} 22$

Blood film comments help to point to a diagnosis; hypersegmented neutrophils and macro-ovalocytes are associated with vitamin B12 and folate deficiency, a uniform macrocytosis with alcohol abuse, target cells with liver disease, and polychromasia with haemolysis.

\section{Neuropsychiatric abnormalities}

Several neuropsychiatric abnormalities have been described in association with vitamin B12 deficiency (including paraesthesia, ataxia, peripheral neuropathy, and memory loss). These may occur in the absence of either anaemia or macrocytosis. Objective signs associated with vitamin B12 deficiency include impaired vibration, touch, pain, and position sense, together with an abnormal gait. ${ }^{23}$

\section{Severe oral ulceration}

Several cases of B12/folate/iron deficiency present with severe oropharyngeal ulceration without changes in the blood count $^{2425}$ and respond to vitamin B12 treatment. ${ }^{26}$

\section{Coexisting conditions}

Coexisting conditions such as iron deficiency or thalassaemia trait may mask the development of macrocytosis and, therefore, in patients who have anaemia without a raised MCV, dual conditions should be considered as a possible diagnosis, and vitamin $\mathrm{Bl} 2$ and folate should be measured if the origin of the anaemia cannot be established by initial tests. ${ }^{20}$

GMS contract indicator: none

How should vitamin B12 or folate deficiency be monitored in patients who have or are receiving replacement?

\section{We recommend:}

- Initially, a full blood count after 10-14 days to document the response and after eight weeks to confirm a normal blood count.

- Longterm:

- folate-not necessary unless the cause persists

- vitamin B12-may not be necessary in replaced patients, although annual checks recommended by some.

\section{Monitoring initial treatment}

Vitamin B12 and folate deficiency: full blood count measurement at 10-14 days to document a rise in the haemoglobin and a fall in MCV and a further check after eight weeks to check that the blood count has returned to normal. ${ }^{22}$ No further monitoring is required once a full haematological response is achieved.

\section{Monitoring longterm treatment}

There is very little published guidance on the monitoring of vitamin $\mathrm{B} 12$ or folate deficiency.

Longterm folate replacement is considered unnecessary in most $\operatorname{cases}^{27}$; the deficiency responds to short term treatment unless the cause (for example, malnutrition) persists.

Patients with pernicious anaemia receiving vitamin B12 replacement should by definition not become vitamin B12 deficient and further monitoring would seem unnecessary in most instances, although practices vary and some haematologists would recommend annual full blood counts. There is no obvious merit in repeating vitamin $\mathrm{B} 12$ and folate measurements during replacement unless lack of compliance (specifically folate) is suspected or anaemia recurs.

GMS contract indicator: none

\section{IMMUNOGLOBULINS AND ELECTROPHORESIS/ PARAPROTEIN BANDS (GAS AND WSAS)}

Much of the guidance is taken from the Consensus Conference on Monoclonal Gammopathies, 1998. ${ }^{28}$ Most of the literature on monoclonal gammopathies examines laboratory methods of diagnosis rather than diagnosis and monitoring guidance. However, this conference produced explicit guidance, although stressed that this is a suggested approach. ${ }^{29}$ It must be noted that the concentrations referred to related to the absolute concentration of a monoclonal band. Laboratories that report electrophoresis would be expected to distinguish clearly between a monoclonal (potentially myeloma related) and a polyclonal increase in 


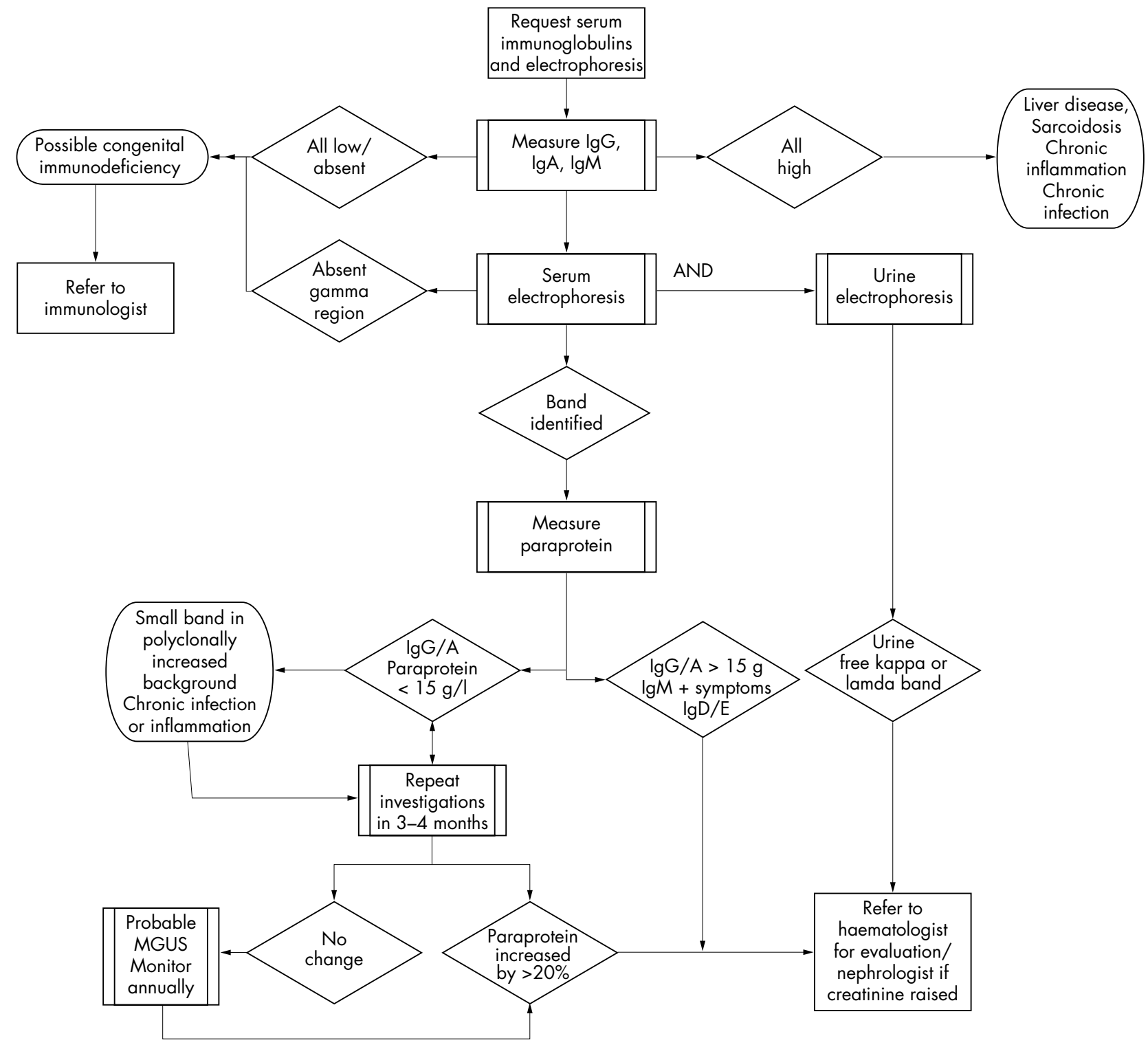

Figure 2 Overview of guidance found on diagnosis and monitoring of paraproteins. MGUS, monoclonal gammopathy of unknown significance.

globulins. This subject is not addressed in the GMS contract. Figure 2 provides an overview of the guidance.

What are the uses of serum immunoglobulins and electrophoresis?

Serum immunoglobulins should be measured and electrophoresis performed:

- As part of the primary screen for suspected plasma cell dyscrasias (myeloma, lymphoma, chronic lymphatic leukaemia, heavy chain disease, amyloid).

- As part of the diagnostic investigation of suspected primary and secondary immunodeficiency; that is, patients with recurrent documented infections.

They are of secondary value in the investigation of:

- Liver disease.

- Connective tissue disease.

- Sarcoidosis.

\section{- Chronic infection.}

\section{Plasma cell dyscrasias and lymphoproliferative} disorders

Electrophoresis followed, where positive, by immunofixation is the most sensitive routine method for the detection of monoclonal proteins. ${ }^{30} 31$ All requests for immunoglobulins should undergo electrophoresis, to exclude possible artefacts caused, in particular, by monoclonal proteins. ${ }^{29}{ }^{32}$

Serum immunoglobulins and electrophoresis, and urine electrophoresis, should be requested in all patients in whom a plasma cell dyscrasia or lymphoproliferative disorder is suspected..$^{30}$ This includes suspected myeloma, Waldenstrom's macroglobulinaemia, lymphoma, chronic lymphocytic leukaemia, and rarer conditions such as amyloid and heavy chain disease. Immunoglobulins alone must not be used to screen for a paraprotein. ${ }^{30}$

Urine electrophoresis should be requested in parallel on an early morning void urine sample or 24 hour collection and quantitated if present. $^{34}$ Standard urine dipsticks will not reliably detect urinary free light chains..$^{30} 323$ 


\section{Immunodeficiency}

Serum immunoglobulins and electrophoresis, and urine electrophoresis, should be requested in all patients in whom immunodeficiency is suspected. Immunodeficiency should be suspected in those who have unusual infections (opportunistic infections), unusual sites of infection (such as the liver, lung, brain abscesses, osteomyelitis), more than two episodes of severe bacterial infection in one year (requiring intravenous antibiotics), one episode of severe infection and recurrent (more than two) minor infections (requiring oral antibiotics). Absent immunoglobulins in a child suggests a congenital antibody deficiency. Normal serum immunoglobulins and electrophoresis do not exclude immunodeficiency, and discussion with an immunologist is appropriate in the clinical scenarios described above if initial investigations are normal. Low immunoglobulins without a paraprotein are also a feature of non-secretory myeloma.

\section{Secondary uses}

Serum immunoglobulins and electrophoresis are of secondary value in the investigation of liver disease: raised IgG in autoimmune hepatitis, raised IgA in cirrhosis (especially as a result of alcohol abuse), and raised IgM caused by primary biliary cirrhosis. These abnormalities are not specific and serum immunoglobulins and electrophoresis should not be used as a primary diagnostic test. Abnormal immunoglobulins may also be seen in sarcoidosis, all connective tissue diseases, chronic bacterial, viral, and fungal infections, and in the immunocompromised, , $^{35}$ but are unlikely to be of benefit in disease monitoring.

Monoclonal bands may be identified in all these conditions, usually on a background of increased polyclonal immunoglobulins. If caused by infection, the bands will disappear after effective treatment. Such bands do not indicate myeloma.

\section{Sample type}

It is essential that serum, not plasma, samples are sent for electrophoresis. Plasma samples will give rise to bands in the $\gamma$ region because of the presence of fibrinogen. Other causes of abnormal bands include raised $C$ reactive protein $\beta$ lipoprotein (pregnancy), transferrin (iron deficiency), and haemolysis (haemoglobin-haptoglobin complexes). ${ }^{36}$

GMS contract indicator: none.

What follow up is required once a patient has been found to have a paraprotein band?

We recommend:

- Initially, three to six month electrophoresis for IgG, IgA, or IgM bands of less than $15 \mathrm{~g}$ /litre without signs or symptoms, and annual electrophoresis thereafter for stable IgG, IgA, or IgM bands of less than $15 \mathrm{~g} /$ litre, where there are no accompanying indicators for a plasma cell dyscrasia.

- Referral to a haematologist for IgA or IgG bands $>15 \mathrm{~g} /$ litre or IgG, IgA, or IgM bands $<15 \mathrm{~g}$ /litre with accompanying indicators for a plasma cell dyscrasia and other immunoglobulin bands (IgE/D: rare).

Relevant accompanying tests after the initial identification of a monoclonal band include: full blood count, calcium, and renal function.

Monoclonal gammopathy of unknown significance (MGUS) or "benign paraproteinaemia" is not uncommon. It has an approximately 60 times greater incidence than myeloma ( $1 \%$ over age of 50 , rising to $3 \%$ over 70 years $^{36}$ ). Approximately $1.5 \%$ of people with MGUS develop myeloma, amyloid, or Waldenstrom's disease, annually, although most die of other diseases. ${ }^{33}$

The paraprotein peak is usually less than $25 \mathrm{~g} / \mathrm{litre}$, although quoted thresholds vary: the UK Myeloma Forum describes MGUS as being characterised by IgA concentrations below $10 \mathrm{~g} /$ /itre and $20 \mathrm{~g} /$ litre for IgG. ${ }^{37}$ Therefore, there is no absolute recommended threshold, although monitoring an existing band as described will detect rises in concentration and therefore appropriateness for referral. Concentrations in myeloma vary, and the clinical decision as to whether or not treatment is required is based on other indicators of disease activity. The primary care threshold recommended is based on referral rather than diagnostic criteria.

Bence Jones proteins (free light chains) in the urine are usually absent or present only at low concentrations $(<50 \mathrm{mg} /$ litre $)$ and no other features of myeloma are present. Twenty five per cent of patients with MGUS have a paraprotein associated with suppression of the other immunoglobulin classes. ${ }^{33}$

Annual serum protein electrophoresis and quantitation by densitometry without need for further immunofixation is recommended for patients with no features of plasma cell dyscrasia (for example, anaemia, bone fracture or pain located in bone, suppression of other immunoglobulin classes, and renal impairment) and a band of $<15$ g/litre. ${ }^{29} 30$

Patients with bands of between 15 and $25 \mathrm{~g} /$ litre are recommended to have additional investigations, including urine electrophoresis. ${ }^{29}$ Depending on local practices, many of these patients will be referred to a haematologist when the band is discovered.

Patients with bands of more than 25 g/litre are more likely to have myeloma, require more detailed initial assessment, including skeletal studies, ${ }^{33}$ and would normally be referred to a haematologist.

GMS contract indicator: none.

\section{HELICOBACTER PYLORI (CAMM, MAG, AND JR)}

These questions and answers make recommendations about when and how primary care should test for Helicobacter pylori. This guidance is based on evidence discussed in detail in other national dyspepsia guidelines available-NICE, the Scottish Intercollegiate Guideline Network, and the Health Protection Agency-therefore, referencing is limited because much of the information can be found in their publications or at their websites. ${ }^{38-41}$ This subject is not addressed in the GMS contract.

\section{Who should I test for $H$ pylori?}

\section{We recommend testing:}

- Patients with a past history of gastric ulcer (GU) or duodenal ulcer (DU) who have not previously been tested.

- Patients with uncomplicated, uninvestigated dyspepsia that is not responsive to lifestyle changes, antacids, $\mathrm{H}_{2}$ antagonists, or proton pump inhibitors (PPIs)..$^{42-46}$

- Patients using non-steroidal anti-inflammatory drugs with diagnosed or previous GU. ${ }^{38}$

Eradication of $H$ pylori in patients with DU and GU cures the ulcer and prevents recurrence. ${ }^{3847}$

In patients using non-steroidal anti-inflammatory drugs, $H$ pylori eradication reduces the risk of the first occurrence of a GU and reduces recurrence of a GU. ${ }^{38} 47$

Symptoms will improve naturally in $36 \%$ of patients with uninvestigated dyspepsia, $7 \%$ will improve as a result of 
eradication treatment, and in 57\% substantial symptoms will remain over a three to 12 month period. ${ }^{46}$

\section{Which non-invasive helicobacter test should I use?}

We recommend the urea breath test or stool antigen test.

The most accurate test (95\% specificity and sensitivity) is the urea breath test, which detects current infection. ${ }^{48}$ This is available on prescription (FP10 BNF section 1.3.1). It requires the patient to swallow a capsule and requires a breath sample at 0 and 20 minutes. This will need to be supervised by a trained individual. The 2003 cost of the breath test was $£ 19.00$, including nurse time.

The stool antigen test also detects current infection and is very accurate, but there have been fewer studies on its accuracy than for the breath test. ${ }^{48}{ }^{49}$ (Current information indicates a specificity and sensitivity of $92-96 \%$ and $93-97 \%$, respectively, depending on the kit manufacturer.) It is a laboratory based test. The patient provides a pea sized piece of stool in the usual laboratory stool pot. No practice nurse time is needed. Specimens should be refrigerated after collection. The 2003 cost of the stool test, including nurse time, is £11.00.

The patient must not take proton PPIs or antibiotics for at least two weeks (ideally four) before, or $\mathrm{H}_{2}$ antagonists for 24 hours before, these tests because these drugs suppress the organism and can lead to false negative results. ${ }^{50-52}$

Laboratory based and near patient blood tests are not as accurate $^{4853}$ (sensitivity and specificity of $88 \%-92 \%$ ). They should only be used if no other tests are available. This test detects antibody to helicobacter. A positive serology result can mean that ${ }^{54}$ :

- The patient is infected at the time of the test.

- The patient had an infection earlier that has resolved, either spontaneously or after specific treatment.

- The test is detecting non-specific, crossreacting antibodies.

\section{What do I do if I find that my patient has helicobacter?}

We recommend that the bacterium is eradicated using triple treatment with a PPI or ranitidine bismuth citrate plus two antibiotics. The importance of compliance with treatment should be stressed.

Analysis of randomised controlled trials by NICE has shown that the most cost effective regimen is PPI once daily plus metronidazole $400 \mathrm{mg}$ twice daily and clarithromycin $250 \mathrm{mg}$ twice daily. ${ }^{38}$

Other combinations of acid reducing agent (PPI or ranitidine bismuth citrate) in combination with two antibiotics may be used as shown in table 1 .

However, because amoxicillin is inactivated at acid $\mathrm{pH}$ PPI twice daily must be used with this drug, and a higher dose of clarithromycin is needed in other combinations. $^{38}$

Table 1 Antibiotics for use in eradicating helicobacter

\begin{tabular}{ll}
\hline Antibiotic & Regimen \\
\hline Amoxicillin & $1 \mathrm{~g} \mathrm{BD}$ \\
Oxytetracycline & $500 \mathrm{mg} \mathrm{QDS}$ \\
Clarithromycin & $500 \mathrm{mg} \mathrm{BD}$ \\
Metronidazole & $400 \mathrm{mg} \mathrm{BD}$ \\
\hline
\end{tabular}

$B D$, twice daily; QDS, four times a day

\section{Do I retest for helicobacter after treatment?}

No, as long as compliance with treatment is good this is unnecessary in most patients with dyspepsia. Only retest in:

- Patients who have DU and are still symptomatic.

- Patients with maltoma (very rare and should be under the care of a specialist).

About $40 \%$ of patients will be asymptomatic after treatment. ${ }^{46}$

In the $60 \%$ with ongoing symptoms of dyspepsia, do not retest unless symptoms suggest that you do. Treat as functional dyspepsia and prescribe PPI or $\mathrm{H}_{2}$ antagonists. The addition of further tests and second line eradication greatly increases costs and there are no reliable data to model further reductions either in risk of infection or dyspepsia symptoms. ${ }^{38}$

\section{Which test should I use if I need to retest?}

We recommend the urea breath test or stool antigen test. PPIs and antibiotics should be stopped for at least two, or preferably four, weeks. ${ }^{50-5255}$

The urea breath test is the most accurate test after treatment ${ }^{49}$ 54; monoclonal stool antigen tests may also be used. ${ }^{49}$

Serology should not be used after treatment because it may detect persisting antibodies. ${ }^{48} 54$

\section{When should I refer patients for endoscopy to diagnose helicobacter?}

We recommend that patients who have complied with two courses of eradication treatment and are still helicobacter positive or patients who are allergic to penicillin and have received clarithromycin and/or metronidazole for other infections should be referred for endoscopy.

Resistance is more common if patients have had courses of clarithromycin or metronidazole for helicobacter or other infections. ${ }^{56}$ When patients are hypersensitive to the recommended antibiotics this reduces the treatment options. In these more complicated scenarios, clinicians should contact their local microbiologist and discuss the possibility of antibiotic susceptibility testing via the helicobacter reference laboratory at the Health Protection Agency.

\section{ACKNOWLEDGEMENTS}

We are most grateful to Mrs S Richardson for typing this manuscript and to the following people who kindly reviewed the work and added valuable comments, in addition to those of the steering group: Professor IS Young (Association of Clinical Biochemists); Dr R Gama, Dr R Herriot (Association of Clinical Pathologists); Dr AJ Mifsud (Association of Medical Microbiologists); Dr E Logan (British Society for Haematology); Dr R Neal, Dr P Hannaford (Royal College of General Practitioners); Dr A Duncan, Dr E Farish, Dr D St J O'Reilly, Dr C Tait, Professor KG Kerr, Dr A Provan (Royal College of Pathologists); Dr A Wierzbicki (Heart UK); and to the other council members of these associations and colleges who assisted in recruiting reviewers.

This work was supported (in alphabetical order) by the Association of Clinical Biochemists, Association of Clinical Pathologists, Association of Medical Microbiologists, British Society for Haematology, Royal College of General Practitioners, Royal College of Pathologists, and the Sowerby Centre for Health Informatics in Newcastle, representatives of whom have contributed to the reviewing process. The opinions stated are however those of the authors.

\section{Authors' affiliations}

W S A Smellie, Department of Pathology, Bishop Auckland Hospital General Cockton Hill Road, Bishop Auckland, County Durham DL14 6AD, UK 
D Wilson, D I Finnigan, PRODIGY, Sowerby Centre for Health Informatics at Newcastle, Bede House, All Saints Business Centre, Newcastle upon Tyne NE1 2ES, UK

C A M McNulty, Health Protection Agency Primary Care Unit, Microbiology Department, Gloucester Royal Hospital, Great Western Road, Gloucester GL1 3NN, UK

M J Galloway, Department of Haematology, E Floor Haematology Office, Sunderland Royal Hospital, Kayll Road, Sunderland SR4 7TP, UK

G A Spickett, Department of Immunology, Royal Victoria Infirmary,

Newcastle upon Tyne NE1 4LP, UK

D A Bareford, Department of Haematology, City Hospital, Dudley Road, Birmingham, West Midlands B18 7QH, UK

M A Greig, Department of Microbiology, St Richard's Hospital, Spitalfield Lane, Chichester, West Sussex PO19 4SE, UK

J Richards, Microbiology Department, Norfolk and Norwich Hospital, Bowthorpe Road, Norwich NR2 3TX, UK

\section{REFERENCES}

1 Smellie WSA, Finnigan DI, Wilson D, et al. Best practice in pathology. Methodology for constructing guidance. J Clin Pathol 2005;58:249-53.

2 NCEP. Detection, evaluation and treatment of high blood cholesterol in adults (adult treatment panel III). Washington: National Institutes of Health, National Heart Lung and Blood Institute, 2002.

3 SIGN. Lipids and the primary prevention of coronary heart disease, 40. Edinburgh: Scottish Intercollegiate Guideline Network, 1999.

4 NSF. National service framework for coronary heart disease. London: Deptartment of Health, 2000.

5 University of Michigan. Screening and management of lipids. Ann Arbor: University of Michigan Health System, 2000;(MI).

6 National Committee on Cardiac Care. Lipids. Singapore: Singapore Ministry of Health, 2001

7 Mclntosh A, Hutchinson A, Feder G, et al. Clinical guidelines and evidence review for type 2 diabetes: lipids management. University of Sheffield, 2002. (http://www.shef.ac.uk/guidelines) last accessed on 11 June, 2004.

8 HPS. MRC/BHF heart protection study of cholesterol lowering with simvastatin in 20,536 high-risk individuals: a randomised placebo-controlled trial. Lancet 2002;360:7-22

9 VHA/DoD. Clinical practice guideline for the management of dyslipidaemia in primary care. Washington (DC): US Department of Defense: Department of Veterans Affairs, 2001.

10 De Backer G, Ambrosioni E, Borch-Johnsen K, et al. European guidelines on cardiovascular disease prevention in clinical practice. Third joint task force of European and other societies on cardiovascular disease prevention in clinical practice. Eur Heart J 2003;24:1601-10.

11 Reynolds TM, Twomey P, Wierzbicki AS. Accuracy of cardiovascular risk estimation for primary prevention in patients without diabetes. J Cardiovasc Risk 2002;9:183-90.

12 American Heart Association recommendations regarding public screening for measuring blood cholesterol. Washington: National Heart, Lung and Blood Institute National Institutes of Health, 1995:95-3045.

13 Pasternak RC, Smith SC Jr, Bairey-Merz CN, et al. ACC/AHA/NHLBI clinical advisory on the use and safety of statins. J Am Coll Cardiol 2002;40:567-72.

14 PRODIGY. Hyperlipidaemia, 2003. (http://www. prodigy.nhs.uk) last accessed 5 February, 2004.

15 UKMI. Drug monitoring requirements in primary care. London, Croydon PCT and London-South Thames Medicine Information Service UK Medicines Information Service, 2002 (http://www.ukmi.nhs.uk) last accessed, 2004

16 Law MR, Wald NJ, Rudnicka AR, et al. Quantifying effect of statins on low density lipoprotein cholesterol, ischaemic heart disease, and stroke: systematic review and meta-analysis. BMJ 2003;326:1423.

17 NICE. Management of type 2 diabetes: management of blood pressure and blood lipids. London, National Institute for Clinical Evidence, 2002 (http:// www.nice.org.uk) last accessed 5 February, 2004.

18 Permeated M, Fernery RE. Monitoring drug treatment. BMJ 2003:327:1179-81

19 CSM. HMG Coal reductive inhibitors (statins) and myopathy. Current Problems in Pharmacovigilance 2002;28:8-9.

20 Lancet, Rapoport A. Macrocytosis. In: Black ER, Bordley DR, Tape TG, et al. Pennsylvania: American College of Physicians, 1999:585-95.

21 PRODIGY. Anaemia, macrocytic, 2003 (http://www.prodigy.nhs.uk)

22 Schrier S. Diagnosis and treatment of vitamin B12 and folic acid deficiency UptoDate.com (http://www.utdol.com) (subscription website) last accessed 26 January, 2004.

23 Stabler SP, Allen RH, Savage DG, et al. Clinical spectrum and diagnosis of cobalamin deficiency. Blood 1990;76:871-81.

24 Porter SR, Scully C, Flint S. Hematologic status in recurrent aphthous stomatitis compared to other oral disease. Oral Surg Oral Med Oral Pathol 1988:66:41-4.

25 Field EA, Speechly JA, Rugman FR, et al. Oral signs and symptoms in patient with undiagnosed vitamin B12 deficiency. J Oral Pathol Med 1995;24:468-70.
26 Wray D, Ferguson MM, Mason DK, et al. Recurrent aphthae: treatment with vitamin B12, folic acid, and iron. BMJ 1975;2:490-3.

27 Drugs used in megaloblastic anaemias. In: British national formulary, 2003. London: British Medical Association and Royal Pharmaceutical Society of Great Britain, 2003:443.

28 Goeken JA, Keren DF. Introduction to the report of the Consensus Conference on Monoclonal Gammopathies. Arch Pathol Lab Med 1998;123:104-5.

29 Kyle RA, Garton JP. Laboratory monitoring of myeloma proteins. Semin Oncol $1986 ; 13: 310-17$

30 Keren DF, Alexanian R, Goeken JA, et al. Guidelines for clinical and laboratory evaluation of patients with monoclonal gammopathies. Arch Pathol Lab Med 1999:123:106-7.

31 Whicher JT, Calvin J, Riches $\mathrm{P}$, et al. The laboratory investigation of paraproteinaemia. Ann Clin Biochem 1987;24:119-32.

32 Keren DF. Procedures for the evaluation of monoclonal immunoglobulins. Arch Pathol Lab Med 1999;123:126-32.

33 Alexanian R, Weber D, Liu F. Differential diagnosis of monoclonal gammopathies. Arch Pathol Lab Med 1999;123:108-13.

34 Graziani M, Merlini G, Petrini C and IFCC Committee on Plasma Proteins, SIBioC Study Group on Proteins. Guidelines for the analysis of Bence Jones protein. Clin Chem Lab Med 2003:41:338-46.

35 Riches P. Paraproteinaemias. In: Marshall WJ, Bangert SK, eds. Clinical biochemistry. Edinburgh: Churchill Livingstone, 1995:493-506.

36 Kyle R. Monoclonal gammopathy of undetermined significance and solitary plasmacytoma. Hematol Oncol Clin North Am 1997;11:71-87.

37 UK Myeloma Forum. Guidelines on the diagnosis and management of multiple myeloma. Br J Haematol 2001;115:522-40.

38 NICE dyspepsia guidance No. 348, 2nd draft out for consultation, 12 December 2003. The management of dyspepsia in primary care. London, NICE (http://www.nice.org.uk/page.aspx?o=20078) last accessed 3 June 2004.

39 Gastroenterologists and Scottish Collegiate Guidelines Network (SIGN) dyspepsia guidelines. (http://www.sign.ac.uk/guidelines/published/ index.html\#Other) last accessed 4 April, 2005.

40 Health Protection Agency. Diagnostic tests-Helicobacter pylori (HP) quick reference guide for primary care (http://www.hpa.org.uk/infections/ topics_az/primary_care_guidance/helicobacter_guide_090305.rtf) last accessed 4 April, 2005

41 Malfertheiner P, Megraud F, O'Morain C, et al. Current concepts in the management of Helicobacter pylori infection. The Maaschricht 2000 consensus report. Aliment Pharmacol Ther 2002;16:167-80.

42 Jones $R$, Tait $C$, Sladen $G$, et al. A trial of a test and treat strategy for Helicobacter pylori positive dyspeptic patients in general practice. Int J Clin Pract 1999:53:413-16.

43 McColl KE, Murray LS, Gillen D, et al. Randomised trial of endoscopy with testing for Helicobacter pylori compared with non-invasive $\mathrm{H}$. pylori testing alone in the management of dyspepsia. BMJ 2002;324:999-1002.

44 Lassen AT, Pedersen FM, Bytzer P, et al. Helicobacter pylori test-and-eradicate versus prompt endoscopy for management of dyspeptic patients: a randomised trial. Lancet 2000;356:455-60

45 Chiba N, Van Zanten SJ, Sinclair P, et al. Treating Helicobacter pylori infection in primary care patients with uninvestigated dyspepsia: the Canadian adult dyspepsia empiric treatment-Helicobacter pylori positive (CADET-Hp) randomised controlled trial. BMJ 2002;324:1012-16.

46 Moayyedi P, Soo S, Deeks J, et al. Systematic review and economic evaluation of Helicobacter pylori eradication treatment for non-ulcer dyspepsia. BMJ 2000;321:649-64.

47 Ford A, Delaney B, Foreman D, et al. Eradication therapy for peptic ulcer disease in Helicobacter pylori positive patients (Cochran review). In: The Cochrane Library. Chichester, UK: John, Wiley \& Son Ltd, 2004;7.

48 Vaira D, Vakil N. Blood, urine, stool, breath, money, and Helicobacter pylori. Gut 2001;48:287-9.

49 Gisbert JP, Pajares JM. Diagnosis of helicobacter infection to stool antigen determination: a systematic review. Am J Gastroenterol 2001;96:2829-38.

50 El-Nujumi A, Hilditch TE, Williams $C$, et al. Current or recent proton pump inhibitor therapy markedly impairs the accuracy of the urea breath test. Eur J Gastroenterol Hepatol 1998; 10:759-64.

51 Vaira E, Vakil N, Menegatti $M$, et al. The stool antigen test for detection of Helicobacter pylori after eradication therapy. Ann Intern Med 2002;136:280-7

52 Graham DY, Opekun AR, Jogi $M$, et al. False negative urea breath tests with $\mathrm{H} 2$ receptor antagonists: interaction between Helicobacter pylori density and pH. Helicobacter 2004:9:1083-9.

53 Laheij RJ, Straatman H, Jansen JB, et al. Evaluation of commercially available Helicobacter pylori serology kits: a review. J Clin Microbiol 1998;36:2803-9.

54 McNulty C, Teare L, Owen R, et al. Test and treat for dyspepsia-but which test [editorial]? BMJ 2005;330:105-6.

55 Burette A. How and when to test or retest for H. pylori. Acta Gastroenterol Belg 1998:61:336-43.

56 Kist M, Glocker E, Wolf B, et al. ResiNet-a nationwide German sentinel study on development and risk factors of antimicrobial resistance in Helicobacter pylori [abstract 16.10]. Helicobacter 2003;8:465. 